\title{
CONSTANT MEAN CURVATURE SURFACES WITH BOUNDARY IN EUCLIDEAN THREE-SPACE
}

\author{
By \\ Rafael López ${ }^{1}$
}

\section{Introduction and Statements of Results}

The study of the structure of the space of constant mean curvature compact surfaces with boundary in Euclidean space $\boldsymbol{R}^{3}$ is the focus of a number of authors. Even in the simplest case, when the boundary is a circle, little is known. For example, if the radius of the circle is $r>0$, a necessary condition due Heinz [5] about the mean curvature $H$ is that $|H| \leq 1 / r$. In this case, the only known examples are the planar disc $(H=0)$, the two corresponding spherical caps of radius $1 /|H|$ if $H \neq 0$, and some non-embedded examples of Kapouleas [8] with genus bigger than two. Also, if the immersion is an embedding, Koiso [9] proved that if the surface is included in one of the two halfspaces determined by the plane containing the circle, then the surface inherits the symmetries of its boundary, and so, the surface is a spherical cap or a planar disc. Recently, Alías, Palmer and the author have proved that the umbilical examples are the only constant mean curvature stable discs with boundary a circle [1].

We will abbreviate a constant mean curvature compact (connected) surface immersed in $\boldsymbol{R}^{3}$ by $c m c$ surface and when we want to emphasize the value $H$ of the mean curvature we will say $H$-cmc surface. Let $\Gamma$ be a Jordan curve in $\boldsymbol{R}^{3}$. We say that $\Sigma$ is a cmc surface with boundary $\Gamma$ when the immersion $\phi: \Sigma \rightarrow \boldsymbol{R}^{3}$ maps the boundary of $\Sigma, \partial \Sigma$, onto $\Gamma$ diffeomorphically. Remark that if $H=0$, the minimal surface $\Sigma$ is contained in the convex hull of $\Gamma$, and hence, if the boundary $\Gamma$ is planar, the surface is included in the boundary plane.

When the boundary $\Gamma$ is a circle of radius $r>0$, Brito and Earp [3] characterized the equality $|H|=1 / r$ in the above Heinz condition, that is, they proved that the hemisphere is the only $(1 / r)$-cmc surface bounded by the circle $\Gamma$. This was proved by using a kind of flux formula and the fact that umbilical

\footnotetext{
${ }^{1}$ This work has been supported by DGICYT. No. PB97-0785

Received July 14, 1997

Revised September 29, 1998
} 
points in a non umbilical $\mathrm{cmc}$ surface are isolated. In section 2, we characterize the equality in the Heinz condition for the case that $\Gamma$ is an arbitrary Jordan planar curve but $\Sigma$ is topologically a disc.

THEOREM 1. Let $\Gamma$ be a Jordan planar curve with length $L$ and let $A$ be the area of the planar domain bounded by $\Gamma$. If $H$ is a nonzero number such that $|H|>$ $L / 2 A$, then there are not $H$-cmc surfaces with boundary $\Gamma$. Moreover, if $|H|=$ $L / 2 A$, the only $H$-cmc disc with boundary $\Gamma$ is a hemisphere of radius $1 /|H|$ and $\Gamma$ is a circle of radius $1 /|H|$.

The first part in Theorem 1 is due to Heinz [5]. By the balancing formula (see Lemma 4 below), the equality $|H|=1 / r$ in the circular boundary case can be viewed as the surface is orthogonal to the boundary plane. In Theorem 2, we consider the case the angle between the surface and this plane is constant along the boundary.

THEOREM 2. Let $\Gamma$ be a circle in a plane $P$. Then the only cme surfaces bounded by $\Gamma$ which make constant angle with $P$ along $\Gamma$, are planar discs and spherical caps.

In section 3, we study a special kind of $H$-cmc surfaces in $\boldsymbol{R}^{3}$. We say that $\Sigma$ is a small $H$-cmc surface if $\Sigma$ is a $H$-cmc surface and included in some Euclidean open ball of radius less than $1 /|H|$. Small $H$-cmc surfaces were defined when one considers the parametric Plateau problem for $H$-cmc discs. In [6], Hildebrandt proved that if $\Gamma$ is a Jordan curve included in some ball $B$ of radius $r>0$ and $H$ verifies $|H|<1 / r$, there exists a $H$-cmc disc spanning $\Gamma$ and contained in $B$. Hence, this solution is a small $H$-cmc surface. When the boundary $\Gamma$ is a circle, Barbosa [2] characterized the spherical caps as the only small $H$-cmc surfaces with boundary $\Gamma$.

We will prove the following existence and uniqueness result on small $H$-cmc graphs bounded by convex planar curves.

THEOREM 3. Give a nonzero constant $H$. Let $\Gamma$ be a convex planar closed curve with curvature $\kappa>|H|$, and let $\Omega$ be the bounded planar domain by $\Gamma$. Then there exists a $H$-cmc graph $G$ on $\Omega$ with boundary $\Gamma$. Moreover, $G$ and its reflection $G^{*}$ of $G$ with respect to the boundary plane are the only small $H$-cmc surfaces with boundary $\Gamma$. 


\section{Proofs of Theorems 1 and 2}

We need the following balancing formula, which was proved by Kusner [11] for constant mean curvature embedded surfaces and also holds in the immersed case (see [10, page 484] and [12]).

Lemma 4 (Balancing formula). Let $\Sigma$ be a $H$-cmc surface with planar boundary. Then

$$
\int_{\partial \Sigma}\langle v, a\rangle d s=2 H \bar{A}
$$

where $v$ is the inner conormal along the boundary, $a \in \boldsymbol{R}^{3}$ is a unit normal vector to the boundary plane and $\bar{A}$ is the algebraic area of $\partial \Sigma$.

Here

$$
\bar{A}=-\frac{1}{2} \int_{\partial \Sigma}\left\langle\alpha \wedge \alpha^{\prime}, a\right\rangle d s,
$$

where $\alpha=\alpha(s)$ is a parametrization of $\partial \Sigma$ such that $\left|\alpha^{\prime}\right|=1$. Thus $|\bar{A}|$ is the area $A$ of the planar bounded domain by $\partial \Sigma$.

ProOf OF THEOREM 1. If $\Sigma$ is a $H$-cmc surface with planar boundary $\Gamma$, the balancing formula leads $|H| \leq L / 2 A$. This statement is the same as in [5].

Now, let assume that the equality holds and the surface $\Sigma$ is a disc. Hence, the balancing formula asserts that $v$ is constant and orthogonal to the boundary plane. Let $\phi: \Sigma \rightarrow \boldsymbol{R}^{3}$ denote the immersion and $\sigma$ its second fundamental form. Then if $t$ is a unit tangent vector along $\partial \Sigma, \sigma(t, v)=0$ on $\partial \Sigma$, and thus $t$ and $v$ are principal directions at each boundary point. We endow $\Sigma$ with the Riemann surface structure given by the conformal class of the induced metric $d s^{2}$ by the immersion $\phi$. Consider the Hopf differential on $\Sigma$

$$
h(d z)^{2}=\left\langle\phi_{z}, N_{z}\right\rangle(d z)^{2},
$$

where $z$ is a local isothermal parameter and $N$ is the Gauss map. Since $\Sigma$ is a constant mean curvature surface in Euclidean space, $h(z)(d z)^{2}$ is holomorphic (see [7, page 137]). Thus, umbilical points of $\phi$ are isolated unless $\phi$ is a totally umbilical immersion.

If $z=u+i v$ is a local isothermic parameter, the Hopf differential is given by

$$
h(d z)^{2}=-\frac{1}{4}(e-g-2 i f)(d z)^{2},
$$


where $\{e, f, g\}$ represent the coefficients of $\sigma$ with respect to the basis $\left\{\phi_{u}, \phi_{v}\right\}$. We can suppose that the immersion $\phi$ is defined in the unit complex disc $D=$ $\{z=u+i v ;|z| \leq 1\}$ and let choose polar coordinates $z=r e^{i \theta}$. Then

$$
\sigma\left(\phi_{r}, \phi_{\theta}\right)=-\frac{r}{2} \sin (2 \theta)(e-g)+r \cos (2 \theta) f
$$

On the other hand, we consider on $D$ the holomorphic map given by $\mu(z)=$ $z^{2} h(z)$, whose imaginary term $\mathfrak{I}(\mu)$ is given by

$$
\mathfrak{I}(\mu)=-\frac{1}{4} r^{2}(e-g) \sin (2 \theta)+\frac{1}{2} r^{2} f \cos (2 \theta)=\frac{r}{2} \sigma\left(\phi_{r}, \phi_{\theta}\right) .
$$

Therefore, the imaginary part of $\mu(z)$ vanishes along the boundary of $D: 2 \mathfrak{J}(\mu)=$ $\sigma(t, v)=0$ along $\partial D$. By using the maximum principle for the harmonic function $\mathfrak{I}(\mu), \mathfrak{I}(\mu) \equiv 0$ on $D$. Then, since $\mu(z)$ is holomorphic, $\mu$ is a constant real number. Thus $h(z)=\mu z^{-2}$ has a pole at $z=0$, unless the constant $\mu$ is zero. From the holomorphy of $h, \mu$ must be zero. Therefore $h=0$ on $D$ and $\Sigma$ is a totally umbilical surface. Consequently, $\Sigma$ is a spherical cap. Since $\Sigma$ intersects orthogonally to the boundary plane, $\Sigma$ is a hemisphere.

Proof of TheOrem 2. After a homothety, we assume the plane $P$ is given by $\left\{(x, y, 0) \in \boldsymbol{R}^{3}\right\}$ and $\Gamma$ is a circle of radius 1 with center at the origin. Let $\Sigma$ be a $H$-cmc surface with boundary $\Gamma$. If $H=0, \Sigma$ is a minimal surface and the maximum principle for cmc surfaces (see Lemma 5 below) concludes the surface is the planar disc bounded by $\Gamma$. Next suppose that $H$ is a nonzero number. Let $N$ be the Gauss map of $\Sigma$. Since the surface makes constant angle with $P$ along $\Gamma$, the function $\langle v, a\rangle$ is constant, where $v$ is the inner conormal along $\Gamma$ and $a$ denotes the vector $(0,0,-1)$. We choose an orientation on $\Gamma$ such that $\left\{\phi_{*}(t), v, N\right\}$ and $\left\{\phi_{*}(t), \phi, a\right\}$ are positively oriented orthonormal basis, where $\phi$ is the immersion of $\Sigma$ in $\boldsymbol{R}^{3}$, and $\phi_{*}(t)$ is a unit tangent field along $\Gamma$. By the balancing formula, the constant function $\langle v, a\rangle$ coincides with $H$. Then

$$
\langle N, \phi\rangle=-\left\langle N \wedge \phi_{*}(t), a\right\rangle=-\langle v, a\rangle=-H,
$$

and hence $\langle N, a\rangle$ is also constant. Since $N=\langle N, a\rangle a+\langle N, \phi\rangle \phi$ on $\partial \Sigma$, the derivative of $N$ along $\partial \Sigma$ is given by $N^{\prime}=\langle N, \phi\rangle \phi_{*}(t)$. Thus, along the boundary $\partial \Sigma$, it holds

$$
\begin{gathered}
\sigma(t, t)=-\left\langle N^{\prime}, \phi_{*}(t)\right\rangle=-\langle N, \phi\rangle=H . \\
\sigma(t, v)=-\left\langle N^{\prime}, v\right\rangle=0 . \\
\sigma(v, v)=2 H-\sigma(t, t)=H .
\end{gathered}
$$


These equalities say us that any point of $\partial \Sigma$ is umbilical. Since the surface has constant mean curvature and the boundary is a set of non-isolated umbilical points [7], then the surface is totally umbilical. Hence, $\Sigma$ is a spherical cap.

\section{Small $H$-cmc Surfaces}

This section deals with small $H$-cmc surfaces with convex planar boundary and we could summarize the main result (Theorem 3) by saying that they are graphs (and so topologically discs) if provided the mean curvature $H$ is small enough. In order to prove this result, we shall make use of a basic tool: the maximum principle for cmc surfaces. Let $\Sigma_{1}, \Sigma_{2}$ be two oriented surfaces in $\boldsymbol{R}^{3}$ and $p$ a common interior point. We say that $\Sigma_{1}$ touches $\Sigma_{2}$ from inside at $p$ if they are tangent at $p$, the orientations in both surfaces agree in $p$ and $\Sigma_{1}$ lies above $\Sigma_{2}$ in a neighborhood of $p$ with respect to the coordinate system given by the tangent plane in $p$ and the unit normal in $p$. Then the maximum principle can be stated as follows (see, for instance, [9]]):

Lemma 5 (Maximum principle). Let $\Sigma_{i}$ be $H_{i}$-cmc surfaces in $\boldsymbol{R}^{3}, i=1,2$, such that $0 \leq H_{1} \leq H_{2}$. If $\Sigma_{1}$ touches $\Sigma_{2}$ at a point $p$ from inside, then $H_{1}=H_{2}$ and $\Sigma_{1}$ and $\Sigma_{2}$ coincide in some neighbourhood of $p$.

In the literature, the classical existence result of $\mathrm{cmc}$ graphs is due to Serrin [13] (see also Theorem 16.11 in [4]) and it can be stated as follows. Let $\Omega$ be a convex planar domain and let $H$ be a nonzero number such that $|H| \leq \kappa / 2$, where $\kappa$ denotes the curvature of the boundary of $\Omega$. Given a Jordan curve $\Gamma$ with single-valued projection on $\partial \Omega$, there exists a $H$-cmc graph defined over $\Omega$ spanning $\Gamma$ (see [12] for another existence result of $H$-cmc graphs). When $\Gamma$ coincides with $\partial \Omega$, it is possible to improve the hypothesis on $H$ obtaining the first part of Theorem 3.

Proof of THE FIRST PART OF TheOREM 3. We have to find a solution of the Dirichlet problem

$$
\begin{cases}\operatorname{div} \frac{\nabla u}{\sqrt{1+|\nabla u|^{2}}}=2 H & \text { on } \Omega, \\ u=0 & \text { on } \partial \Omega .\end{cases}
$$

This can be achieved using a continuity method (see [4, Theorem 13.8]) if we can obtain a priori $C^{1}(\bar{\Omega})$-estimate independent of $H$ for each solution $u$ of $(1)$ with 
$0 \leq H \leq c$, where $c$ is a fixed real number such that $c<\kappa$. Standard theory of quasilinear equations [4, section 11.3] assures that it is sufficient to get a priori $C^{0}(\bar{\Omega})$-estimate and $C^{1}$-estimate along $\partial \Omega=\Gamma$ of $u$.

For $H=0$, we have the trivial solution $u \equiv 0$. Then it follows from the implicit function theorem that there exist solutions of (1) with $0 \leq H \leq c_{0}$ for an enough small number $c_{0}$. For every solution $u$ of (1) with $c_{0} \leq H \leq c$, the height lemma for graphs due to Serrin [14] implies that

$$
|u| \leq \frac{1}{|H|} \leq \frac{1}{c_{0}}
$$

So we obtain a priori $C^{0}(\overline{\mathbf{\Omega}})$-estimate of $u$.

The $C^{1}$-estimates of $u$ along the boundary $\Gamma$ are obtained provided we are able to establish a priori estimates of the slope of the graph $G$ of $u$ along the boundary (see [12, Corollary 4] for an example in the same context). This estimate will be accomplished by the technique of barriers. Without loss of generality, we assume that $P=\left\{(x, y, 0) \in \boldsymbol{R}^{3}\right\}$ is the boundary plane and $G$ is contained in the halfspace $\{z \geq 0\}$. Since $\Gamma$ is compact, we can take positive numbers $r_{1}, r_{0}$ such that

$$
\kappa>\frac{1}{r_{1}}>\frac{1}{r_{0}}>|H|
$$

Let $K$ be a hemisphere of radius $r_{0}$ such that $K \subset\{z \geq 0\}$ and $\partial K \subset P$.

We consider a circle $C_{1}$ of radius $r_{1}$ in $P$ such that $\Omega$ is included in the planar domain $\Omega_{1}$ bounded by $C_{1}$. This is possible because $r_{1}>1 / \kappa$ and so, there exists a circle $C_{1}$ of radius $r_{1}$ that contains $\Gamma$ in its interior and with the property that $C_{1}$ rolls along $\Gamma$ touching every point of $\Gamma$. After a horizontal translation respect to $P$, we put $K$ such that $\partial K$ is a concentric circle with $C_{1}$.

We move upwards $K$ to do not intersect $G$. Next, we move it down. Since the mean curvature of $K$ is $1 / r_{0}$ with the orientation pointing inwards and $1 / r_{0}>$ $|H|$, the maximum principle assures that we can come back to the original position of $K$ without touching $G$. Whence $G$ lies below $K$ with respect to the positive vertical direction.

For each $t>0$, let $K_{t}=(K+(0,0,-t)) \cap\{z \geq 0\}$, where $K+(0,0,-t)$ denotes the vertical translation of $K$ by the vector $(0,0,-t)$. Since $\partial K$ and $\Gamma$ are disjoint compact sets, we can slightly move down $K$ and not intersect the graph $G$ until $\partial K_{t}$ touches $C_{1}$. This is possible because in the case that $K_{t_{0}}$ should intersect $G$ at the first time $t_{0}>0$, this would occur at an interior point of $G \cap K_{t_{0}}$ since 
$\Gamma \subset \Omega_{1}$. But at this interior point, the mean curvature of $K_{t_{0}}$ is $1 / r_{0}$ and the maximum principle would give a contradiction because $1 / r_{0}>|H|$.

Let $K_{t_{1}} \subset K$ denote the spherical cap such that $\partial K_{t_{1}}=C_{1}$. Because $t_{1}>0, K_{t_{1}}$ is a $\left(1 / r_{0}\right)$-cmc graph on $\Omega_{1}$. Moreover, the existence of $K_{t_{1}}$ depends only on $r_{0}$ and $r_{1}$, that is, on $\Gamma$ and $H$. In this proof, the role of barrier surface will be played by the cap $K_{t_{1}}$. We move $K_{t_{1}}$ respect to any horizontal direction until to reach $G$. Again, the maximum principle implies that the first intersection point occurs at some boundary point. Now, a "rolling" argument comparing $\partial K_{t_{1}}$ and $\Gamma\left(r_{1}>1 / \kappa\right)$, and the fact that $1 / r_{0}>|H|$ assert that in every point of $\Gamma$, the slope of the graph $G$ is less than the slope of $K_{t_{1}}$. It follows that the (constant) slope of $K_{t_{1}}$ along $C_{1}$ is an upper bound of the slope of $G$ along $\Gamma$. Therefore, we find the $C^{1}$-estimate of $u$ along $\partial \Omega$ that we were looking for. This proves the first assertion of Theorem 3.

In order to prove the second statement of this theorem, we need an auxiliary lemma. For a point $p \in \boldsymbol{R}^{3}$ and a positive constant $r$, let $B(p, r)$ be the Euclidean open ball of radius $r$ and with center at $p$, and $S(p, r)$ the boundary sphere of $\partial B(p, r)$.

LemMa 6. Let $\Gamma \subset R^{3}$ be a planar Jordan curve. If $\Sigma$ is a small H-cmc surface with boundary $\Gamma$, then

$$
\Sigma \subset \bigcap\{B(q, s) ; s \leq 1 /|H|, \Gamma \subset B(q, s)\} .
$$

Proof. We may assume the plane $P$ containing $\Gamma$ is $\left\{(x, y, 0) \in \boldsymbol{R}^{3}\right\}$. Since $\Sigma$ is a small $H$-cmc surface, there exists an Euclidean open ball $B(p, r)$ of radius $r \leq|H|$ containing $\Sigma$ inside.

Let $p_{0}$ be the orthogonally projected point on $P$ of $p$. We can prove $\Sigma \subset$ $B\left(p_{0}, r\right)$ : We may assume $p \neq p_{0}$. Since the distance $d\left(p_{0}, \Gamma\right)$ between $p_{0}$ and $\Gamma$ is less than $d(p, \Gamma)$, it is clear that $\Gamma \subset B\left(p_{0}, r\right)$. Suppose $\Sigma \not \subset B\left(p_{0}, r\right)$. Then there exists a point $p_{t}$ on the segment from $p$ to $p_{0}$ such that $\Sigma$ touches $S\left(p_{t}, r\right)$ from inside at some interior points. The maximum principle combined with the analyticity of surfaces asserts that $\Sigma$ is just a part of $S\left(p_{t}, r\right)$. Then $\Gamma=S\left(p_{t}, r\right) \cap$ $P$ and hence $d(p, \Gamma) \leq d\left(p_{t}, \Gamma\right)=r$. This is a contradiction.

Assume there exists an Euclidean open ball $B(q, s)$ of radius $s \leq 1 /|H|$ such that $\Gamma \subset B(q, s)$ and $\Sigma \not \subset B(q, s)$. Let $q_{0}$ be the orthogonally projected point on $P$ of $q$. It is clear that $\Gamma \subset B\left(q_{0}, s\right)$ and we can prove that $\Sigma \not \subset B\left(q_{0}, s\right)$ by using the maximum principle similarly as above.

When $s \leq r$, it is clear that $\Gamma \subset B\left(q_{0}, r\right)$ and we can prove that $\Sigma \not \subset B\left(q_{0}, r\right)$ : 
We may assume $s<r$. If $\Sigma \subset B\left(q_{0}, r\right)$, there exists $t \in[s, r)$ such that $\Sigma$ touches $S\left(q_{0}, r\right)$ from inside at some interior points. The maximum principle combined with the analyticity of surfaces asserts that $\Sigma$ is just a part of $S\left(q_{0}, t\right)$. Then $\Gamma=S\left(q_{0}, t\right) \cap P \not \subset B\left(q_{0}, s\right)$. This is a contradiction.

Since $\Sigma \subset B\left(p_{0}, r\right)$ and $\Sigma \not \subset B\left(q_{0}, r\right)$, there exists a point $q_{t} \in P$ on the segment from $q_{0}$ to $p_{0}$ such that $\Sigma$ touches $S\left(q_{t}, r\right)$ from inside at some interior points. The maximum principle combined with the analyticity of surfaces asserts that $\Sigma$ is just a part of $S\left(q_{t}, r\right)$. Then $\Gamma=S\left(q_{t}, r\right) \cap P$. This is a contradiction to the fact $\Gamma \subset$ $B\left(p_{0}, r\right) \cap B\left(q_{0}, r\right)$.

When $s>r$, since $\Sigma \subset B\left(p_{0}, s\right)$ and $\Sigma \not \subset B\left(q_{0}, s\right)$, we can also obtain a contradiction by using a similar argument as above.

Accordingly, for any Euclidean open ball $B$ of radius $s \leq 1 /|H|$ such that $\Gamma \subset B, \Sigma$ must be included in $B$.

Now we are in conditions to prove the final part of Theorem 3. Let $P$ be the plane containing $\Gamma$ and assume that $P=\left\{(x, y, 0) \in R^{3}\right\}$. Let $\kappa>0$ be the curvature function of $\Gamma$ and let $\kappa_{0}>0$ be the minimum value of $\kappa$. Put $s=1 / \kappa_{0}$. There exists $q \in P$ such that $\Gamma \subset \overline{B(q, s)} \cap P$. Let $\Sigma$ be a small $H$-cmc surface with boundary $\Gamma$ and let $H$ be with $0<|H|<\kappa$. Then

$$
\Gamma \subset \overline{B(q, s)} \cap P \subset B\left(q, \frac{1}{|H|}\right) \cap P
$$

and, from Lemma 6, we have $\Sigma \subset B(q, 1 /|H|)$.

Move $S(q, 1 /|H|)$ in any direction parallel to $P$ until to touch $\Sigma$, which we continue to denote by $S(q, 1 /|H|)$. By using the maximum principle, there is no point in $\Sigma \backslash \Gamma$ which touches with $S(q, 1 /|H|)$. Then we can carry $S(q, 1 /|H|)$ until it reaches $\Gamma$. Since the radius of the circle $S(q, 1 /|H|) \cap P$ is $1 /|H|>1 / \kappa_{0}=s$, we can move $S(q, 1 /|H|)$ in any direction parallel to $P$ to touch each point of $\Gamma$. Therefore $\Sigma$ is contained in the right cylinder determined by the bounded domain $\Omega \subset P$ by $\Gamma$, specifically,

$$
\Sigma \subset \overline{\boldsymbol{\Omega}} \times \boldsymbol{R} \quad \boldsymbol{\Sigma} \cap(\Gamma \times \boldsymbol{R})=\Gamma .
$$

Let $G$ be the $H$-cmc graph on $\Omega$ with boundary $\Gamma$ whose existence is assured by the first statement of Theorem 3. Then, we have in $\Omega \times \boldsymbol{R}$ two cmc surfaces, $\Sigma$ and $G$, with the same constant mean curvature $H$ and the same boundary $\Gamma=$ $\partial \Omega \times\{0\}$. It remains then to show that $\Sigma$ coincides with $G$ of its reflected $G^{*}$ with respect to $P$. This fact is a uniqueness result that appears in [12] but for the sake of completeness, we do its proof. 
Let $a=(0,0,1)$ and we suppose that $G$ is over $P$. We orient $G$ to have $H>0$ and let $N_{G}$ be its Gauss map. Then $\left\langle N_{G}, a\right\rangle\langle 0$. We move up $G$ to have no intersection with $\Sigma$ (this is possible because $\Sigma$ and $G$ are compact) and next, we move down $G$ to touch $\Sigma$. If there is a contact point (interior or boundary) and because $N_{G}$ points down, then $\Sigma$ agrees with $G$ (maximum principle). Similar arguments work with $G^{*}$. Thus we have two possibilities: $\Sigma$ is $G$ or $G^{*}$ or otherwise, $\Sigma$ is included in the domain of $\boldsymbol{R}^{3}$ bounded $G \cup G^{*}$. In this case, we shall get a contradiction. For that, if we compare the inner conormal vectors $v_{\Sigma}$, $v_{G}$ of $\Sigma$ and $G$ respectively along $\Gamma$, we have the inequality $\left|\left\langle v_{\Sigma}, a\right\rangle\right|\left\langle\left\langle v_{G}, a\right\rangle\right.$ and thus

$$
\int_{\partial \Sigma}\left|\left\langle v_{\Sigma}, a\right\rangle\right|<\int_{\partial G}\left\langle v_{G}, a\right\rangle
$$

But the balancing formula and the fact that the immersion along the boundary $\partial \Sigma$ is a diffeomorphism onto $\Gamma$, we have that

$$
\left|\int_{\partial \Sigma}\left\langle v_{\Sigma}, a\right\rangle\right|=\int_{\partial G}\left\langle v_{G}, a\right\rangle=2 H A
$$

where $A$ is the area of $\Omega$. This is a contradiction with the previous strict integral inequality and this completes the proof of Theorem 3 .

REMARK. As consequence of Theorem 3 we have that given a convex planar closed curve $\Gamma$, Hildebrandt's solutions [6] with boundary $\Gamma$ are graphs if $H$ is small enough.

\section{References}

[1] Alías, L. López, R. and Palmer, B., Stable constant mean curvature surfaces with circular boundary, Proc. A.M.S., 127 (1999), 1195-1200.

[2] Barbosa, J. L., Hypersurfaces of constant mean curvature on $\boldsymbol{R}^{n+1}$ bounded by an Euclidean sphere, Geometry and Topology II, World Scientific Publishing Co. Pte. Ldt., Singapore 1990, 1-9.

[3] Brito, F. and Earp, R., Geometric configurations of constant mean curvature surfaces with planar boundary, Anais da Acad. Bras. de Ciências, 63 (1991), 5-19.

[4] Gilbarg, D. and Trudinger, N. S., Elliptic Partial Differential Equations of Second Order, 2nd edition, Springer-Verlag, Berlin, 1983.

[ 5] Heinz, H., On the nonexistence of a surface of constant mean curvature with finite area and prescribed rectificable boundary, Arch. Rational Mech. Anal., 35 (1969), 249-252.

[6] Hildebrandt, S., On the Plateau problem for surfaces of constant mean curvature, Comm. Pure Appl. Math., 23 (1970), 97-114.

[7] Hopf, H., Differential Geometry in the Large. Lecture Notes in Mathematics, 1000. SpringerVerlag, Berlin, 1983. 
[ 8 ] Kapouleas, N., Compact constant mean curvature surfaces in Euclidean three-space, J. Diff, Geom., 33 (1991), 683-715.

[9] Koiso, M., Symmetry of hypersurfaces of constant mean curvature with symmetric boundary, Math. Z., 191 (1986) 567-574.

[10] Korevaar, N. Kusner, R. and Solomon, B., The structure of complete embedded surfaces with constant mean curvature, J. Diff, Geom., 30 (1989), 465-503.

[11] Kusner, R., Global geometry of extremal surfaces in three-space, Doctoral thesis, University of California, Berkeley, 1985.

[12] López, R. and Montiel S., Constant mean curvature surfaces with planar boundary, Duke Math. J., 85 (1996), 583-604.

[13] Serrin, J., The problem of Dirichlet for quasilinear elliptic equations with many independent variables, Philos. Trans. Roy. Soc. London Ser. A 264 (1969), 413-496.

[14] Serrin, J., On surfaces of constant mean curvature which span a given space curve, Math. Z., 112 (1969), 77-88.

Departamento de Geometría y Topología

Universidad de Granada

18071 Granada. Spain

e-mail: rcamino@ugr.es 